\title{
Pathogenicity of an FAdV-4 isolate to chickens and its genomic analysis ${ }^{* \#}$
}

\author{
Kai-kun MO, Chen-fei LYU, Shang-shang CAO, Xia LI, Gang XING, \\ Yan YAN, Xiao-juan ZHENG, Min LIAO ${ }^{\dagger}$, Ji-yong ZHOU \\ Key Laboratory of Animal Virology of Ministry of Agriculture, Zhejiang University, Hangzhou 310058, China \\ ${ }^{\dagger}$ E-mail: liaomin4545@zju.edu.cn \\ Received Feb. 14, 2019; Revision accepted May 2, 2019; Crosschecked July 9, 2019
}

\begin{abstract}
Fowl adenovirus serotype 4 (FAdV-4) strain SD1511 was isolated from chickens with severe inclusion body hepatitis and hydropericardium syndrome in Shandong Province, China. The isolate was cultured in primary chicken embryo kidney cells. A study of pathogenicity indicated that SD1511 readily infected 7-35-d-old chickens by intramuscular injection and intranasal and oral routes, causing 50\%-100\% mortality. The 35 -d-old chickens suffered more severe infection than 7- and 21-d-old chickens with mortality highest in the intramuscular injection group. The serum from surviving chickens showed potent viral neutralizing capability. The complete genome of SD1511 was sequenced and analyzed. The strain was found to belong to the FAdV-4 cluster with more than $99 \%$ identity with the virulent FAdV-4 strains isolated in China in recent years except for some distinct variations, including deletions of open reading frame 27 (ORF27), ORF48, and part of ORF19. Our findings suggest that SD1511 might be used as a prototype strain for the study of pathogenesis and vaccine development.
\end{abstract}

Key words: Fowl adenovirus serotype 4 (FAdV-4); Pathogenicity; Infection way; Virus neutralization activity; Genome analysis

https://doi.org/10.1631/jzus.B1900070

CLC number: S858.31

\section{Introduction}

Fowl adenoviruses (FAdVs) are ubiquitous in the chicken industry all over the world. They have been grouped into five species, A, B, C, D, and E, and 12 serotypes (Hess, 2000). They are non-enveloped and non-segmented double-stranded DNA viruses with a linear genome approximately $43-45 \mathrm{~kb}$ in

\footnotetext{
Corresponding author

* Project supported by the National Key Technology Research and Development Program of China (No. 2015BAD12B01) and the China Agriculture Research System (No. CARS-40-K13)

\# Electronic supplementary materials: The online version of this article (https://doi.org/10.1631/jzus.B1900070) contains supplementary materials, which are available to authorized users

(i) ORCID: Kai-kun MO, https://orcid.org/0000-0001-7323-9475; Min LIAO, https://orcid.org/0000-0003-3006-9335

(C) Zhejiang University and Springer-Verlag GmbH Germany, part of Springer Nature 2019
}

length, belonging to the genus Aviadenovirus, family Adenovirida (Hess, 2000). FAdVs were thought to be weakly virulent, requiring co-infection with immunosuppressive viruses to cause disease. However, in recent years, knowledge about these viruses has increased, as has their importance with frequent reports of highly virulent strains causing hepatitishydropericardium syndrome (HHS) and adenoviral gizzard erosion (AGE) in poultry (Schachner et al., 2018).

HHS, also known as "Angara disease," was first reported in 3-5-week-old broilers in Angara Goth, Pakistan in 1987 (Jaffery, 1988). HHS is a severe condition that features the accumulation of clear or amber-colored liquid in the pericardial sac, and damages heart tissue. Aside from heart deformities, damage to the livers, lungs, and kidneys has also been 
observed. Intra-nuclear inclusion bodies in the livers are regarded as a typical feature, accompanied by necrotic and dystrophic changes (Mazaheri et al., 1998; Ganesh and Raghavan, 2000). HHS results in the mortality of $50 \%$ to $100 \%$ of unvaccinated birds (Ganesh and Raghavan, 2000).

Reports of HHS have increased around the world in recent years. The chicken industries of many countries, including India (Mittal et al., 2014), Canada (Ojkic et al., 2008), Hungary (Kaján et al., 2013), Republic of Korea (Choi et al., 2012), and Japan (Mase et al., 2012), have suffered considerable economic losses. Earlier outbreaks in China were also confirmed ( $\mathrm{Li}$ et al., 2010). However, since 2012, clinical cases of HHS have increased and spread all over the country, with most observed in 3-5-week-old broilers. The etiology of most of these cases was identified as serotype 4 of the FAdV C species (FAdV-4) (Zhao et al., 2015). Compared with previous HHS cases caused by FAdVs, the outbreaks of FAdV-4 in northern China in 2015 contributed to great economic loss for the poultry industry. Reported cases of HHS have been increasing significantly in many provinces of China, including Shandong, Hubei, Jiangsu, Anhui, Jiangxi, Henan, and Zhejiang (Li et al., 2017).

In this study, an FAdV-4 strain, SD1511, was isolated from a recent outbreak in Shandong, China. The pathogenicity of SD1511 was investigated in different chicken age groups by several infectious routes. The full genome of the isolate was then sequenced and compared with FAdV-4 strains isolated recently in China and reference strains originating from outside China.

\section{Materials and methods}

\subsection{Sample collection and experimental animals}

Liver tissue samples were collected from birds presenting with fluid accumulation in the pericardial sac and an enlarged discolored liver with foci of hemorrhage and/or necrosis. The birds originated from a chicken farm in Shandong Province.

Specific-pathogen-free (SPF) white leghorn chickens and embryonated eggs were purchased from the Shennong Company (Zhejiang, China). Chickens were maintained in SPF isolators under negative pressure. The animal experiment was approved by the
Committee on the Ethics of Animal Care and Use at Zhejiang University (Hangzhou, China) (No. ZJU 20170388).

\subsection{Virus isolation and identification}

The liver tissue samples were homogenized in phosphate-buffered saline (PBS). The homogenates were centrifuged at $12000 \mathrm{r} / \mathrm{min}$ for $10 \mathrm{~min}$ following three freeze and thaw cycles. The supernatants were used for DNA extraction and virus isolation.

For virus isolation, the supernatants were inoculated into the 8-d-old SPF embryonated chicken eggs through the chick chorioallantoic membrane. The chorioallantoic membrane and allantoic fluid were then harvested and passed three times into SPF embryonated chicken eggs. Virus was confirmed by PCR amplification and sequencing. The isolate was named SD1511.

The isolate from embryonated eggs was inoculated into primary chicken embryo kidney (CEK) cells prepared from 15-18-d-old embryonated eggs and cultured in RPMI 1640 medium (Gibco, USA) with $10 \%$ fetal bovine serum.

For median tissue culture infectious dose $\left(\mathrm{TCID}_{50}\right)$ determination, indirect immunofluorescent assay (IFA) was performed to detect the virus-infected cells with antiserum from mouse immunized with the purified hexon protein prepared in our laboratory.

\subsection{PCR detection and sequence analysis}

Total DNA was extracted from either the supernatant of tissue homogenate, or virus isolate products from SPF embryonated chicken eggs or infected CEK cells using the traditional DNA extraction method. The PCR was performed with primer pairs (FAV-Hexon-F: 5'-ATACCAACACGAGCACCAC-3'; FAV-Hexon-R: 5'-TTATCCCTGAACCCGATG-3') designed with the available nucleotide sequences of FAdV-4 strain KR5 (GenBank: HE608152.1). The reactions were performed as follows: $5 \mathrm{~min}$ at $95^{\circ} \mathrm{C}$, followed by 35 cycles at $95^{\circ} \mathrm{C}$ for $30 \mathrm{~s}, 58^{\circ} \mathrm{C}$ for $30 \mathrm{~s}$, and $72{ }^{\circ} \mathrm{C}$ for $15 \mathrm{~s}$, and a final elongation step of $10 \mathrm{~min}$ at $72^{\circ} \mathrm{C}$. PCR products were further subjected to sequencing and analysis by BioSune (Shanghai, China).

\subsection{Genome sequencing and analysis}

Thirty-eight primer pairs (Table S1) were synthesized according to the primers designed by Zhao et al. 
(2015) to amplify the DNA fragments which cover the full length of the isolate genome. All primers were synthesized by Sangon Biotech (Shanghai, China).

PCR was performed using the Phanta SuperFidelity DNA Polymerase (Vazyme Biotech, Nanjing, China). Each $50-\mu \mathrm{L}$ PCR reaction volume consisted of $25 \mu \mathrm{L} 2 \times$ reaction buffer, $1 \mu \mathrm{L}$ polymerase $(10 \mu \mathrm{mol} / \mathrm{L}), 1 \mu \mathrm{L}$ total DNA from SD1511-infected CEK cells, $1 \mu \mathrm{L}$ dNTPs $(10 \mathrm{mmol} / \mathrm{L}), 1 \mu \mathrm{L}$ of each primer $(10 \mu \mathrm{mol} / \mathrm{L})$, and nuclease-free water up to $50 \mu \mathrm{L}$. The reaction was performed as follows: 5 min at $95^{\circ} \mathrm{C}$, followed by 35 cycles of $20 \mathrm{~s}$ at $95^{\circ} \mathrm{C}$, $30 \mathrm{~s}$ at $52-60{ }^{\circ} \mathrm{C}$, and $50 \mathrm{~s}$ at $72{ }^{\circ} \mathrm{C}$, and a final elongation step for $5 \mathrm{~min}$ at $72{ }^{\circ} \mathrm{C}$. $\mathrm{PCR}$ products were examined by electrophoresis using $1.0 \%(0.01 \mathrm{~g} / \mathrm{mL})$ agarose gel and visualized following DuRed staining. The amplified products were then elongated using rTaq polymerase (TaKaRa Biotechnology Co., Ltd., Dalian, China) at $72{ }^{\circ} \mathrm{C}$ for $10 \mathrm{~min}$ and cloned into the pMD18-T vector (TaKaRa) for sequencing.

Sequence assembly of the DNA fragment was done using DNAMAN (Version 9.0) followed by alignment using the Basic Local Alignment Search Tool, a bioinformatics tool available from the National Center for Biotechnology Information (NCBI, http://blast.ncbi.nlm.nih.gov/Blast.cgi).

The sequence of the full-length genome of SD1511 was aligned with homologues of different FAdV species available from GenBank using MEGA software (Version 5.05). To analyze the relationship of the isolate to other FAdVs, phylogenetic trees were constructed using neighbor-joining analysis with 500 bootstrap replicates, and the maximum composite likelihood method in MEGA5.05 software. The phylogenetic datasets for analysis included 8 FAdV-4 isolates and 14 reference strains of FAdV-A, FAdV-B, FAdV-D, FAdV-E, Turkey adenovirus 1, Avian adenovirus egg-drop syndrome virus (EDSV), Human adenovirus C, Goose adenovirus 4, and Hemorrhagic enteritis virus.

\subsection{Pathogenicity assessment}

To examine the pathogenicity of SD1511, chickens of three age groups $(7,21$, and $35 \mathrm{~d}$ ) were randomly divided into three groups with at least ten birds in each group. Chickens were inoculated with $3 \times 10^{3.2}$ $\mathrm{TCID}_{50}$ of strain SD1511 via intramuscular (i.m.) or intranasal (i.n.) injection. The control birds in every age group received equal volumes of PBS.

To examine the effects of different infectious routes on FAdV-4 injection, forty 35-d-old SPF chickens were divided into four groups and inoculated with the same virus titer $\left(3 \times 10^{3.2} \mathrm{TCID}_{50}\right)$ via i.m., i.n., or oral (o.r.) injection. One group was left uninfected as the control. Chickens were observed daily until the 40th day post-inoculation (dpi) and the deaths were recorded. Four tissue samples (heart, liver, lung, and kidney) were collected. One part of each of the heart and liver tissue samples was immediately placed into $10 \%$ neutral buffered formalin solution for histological examination, and the remainder was stored at $-80{ }^{\circ} \mathrm{C}$ for detection of viral load.

\subsection{Histopathology and immunohistochemistry examinations}

Samples of heart and liver were fixed in $10 \%$ neutral buffered formalin solution for at least $48 \mathrm{~h}$ at room temperature. The tissues were then sent to Wuhan Servicebio Technology Co., Ltd. (Wuhan, China) for processing including hematoxylin and eosin (H\&E) staining and immunohistochemistry. Immunohistochemistry was performed with a chicken anti-FAdV hyperimmune serum provided by Professor Guo-zhong ZHANG (China Agricultural University, Beijing, China) (Zhao et al., 2015).

\subsection{Detection of viral loads in different tissues}

Viral loads in different tissues from different treatment groups were examined by real-time PCR. Identical weights of each of the four tissue samples (heart, liver, lung, and kidney) from three chickens in each group were collected and homogenized in PBS. The homogenates were then centrifuged at $12000 \mathrm{r} / \mathrm{min}$ for $10 \mathrm{~min}$ following three freeze and thaw cycles. Equal volumes of supernatant were used for DNA extraction. Virus load was detected by real-time PCR using the primer pairs FAdV-H(part)-F (5'-ACTA CACCAACAGCCTCTCG-3') and FAdV-H(part)-R (5'-TTATCCCTGAACCCGATG-3').

Real-time PCR was performed on the 7500 real-time PCR system (Applied Biosystems, CA, USA) in a total volume of $20 \mu \mathrm{L}$ containing AceQ qPCR SYBR Green I Master Mix (Vazyme Biotech, Nanjing, China) as a fluorescent dye. Each reaction 
contained $1 \mu \mathrm{L}$ of the above prepared template DNA and $0.4 \mu \mathrm{L}$ of each primer $(10 \mu \mathrm{mol} / \mathrm{L})$. The cycling program consisted of one cycle at $95{ }^{\circ} \mathrm{C}$ for $30 \mathrm{~s}, 40$ cycles at $95{ }^{\circ} \mathrm{C}$ for $5 \mathrm{~s}$ and $60{ }^{\circ} \mathrm{C}$ for $40 \mathrm{~s}$, with a melting step between 60 and $95^{\circ} \mathrm{C}$. The fluorescence data were collected during the annealing/extension step. All samples were measured in triplicate and the data were analyzed using ABI 7500 software (Version 2.0.6).

\subsection{Detection of virus shedding}

To detect virus shedding, oropharyngeal and cloacal swabs of infected chickens in the i.n. group were collected 2 to 40 dpi and viral DNA was extracted for PCR detection with the primer pairs and the program mentioned above.

\subsection{Serum neutralization assay}

To evaluate the neutralization activity of serum antibody to the FAdV-4 isolate, blood samples were collected at 45 dpi from the surviving chickens of the 7-d-old group infected via the i.n. route, as well as from the parallel control group. The serum samples were prepared by centrifugation at $12000 \mathrm{r} / \mathrm{min}$ for $5 \mathrm{~min}$. For the sera from infected animals, serial dilutions $\left(10^{-1}, 10^{-2}\right.$, and $\left.10^{-3}\right)$ were made in $500 \mu \mathrm{L}$ in PBS. The negative sera collected from uninfected birds were diluted to $10^{-1}$ as negative control and blank control was set with PBS. All serum samples and one PBS control pre-incubated with $0.5 \mathrm{~mL}$ of SD1511 $\left(5 \times 10^{3.2} \mathrm{TCID}_{50}\right)$ for $1 \mathrm{~h}$ at $37{ }^{\circ} \mathrm{C}$ were inoculated into $\mathrm{CEK}$ cells that had been seeded one day before infection. Infected cells were incubated at $37{ }^{\circ} \mathrm{C}$ for $36 \mathrm{~h}$ and the culture supernatant was passed once to a new CEK cell. The cells of the second passage were collected for viral DNA detection by real-time PCR and common PCR.

To further determine the serum neutralizing antibody titer, 100 TCID $_{50}$ of SD1511 was pre-incubated with serial dilutions $\left(10^{-2}, 10^{-3}, 10^{-4}, 10^{-5}, 10^{-6}\right.$, and $10^{-7}$ ) of sera from surviving chickens for $1 \mathrm{~h}$ at $37^{\circ} \mathrm{C}$ prior to infection of CEK cells in 96-well plates. The infected CEK cells were cultured for $72 \mathrm{~h}$ and then detected by IFA with mouse antiserum to recombinant hexon protein prepared in our laboratory. The serum neutralizing antibody titer was calculated by the method of Reed and Muench (Lund et al., 1995). Each dilution series was performed in eight repetitions.
To determine the $\mathrm{TCID}_{50}$ of SD1511, the virus stock collected from infected CEK cell culture was diluted 10-fold, and these dilutions were used to infect the CEK cells in a 96-well plate. Each dilution $(100 \mu \mathrm{L})$ was added to each well of the plate, and each dilution had eight repetitions. The determination and calculation of the $\mathrm{TCID}_{50}$ of SD1511 were the same as described above for the determination of the serum neutralizing antibody titer.

\subsection{Statistical analysis}

Statistical analysis was performed by GraphPad Prism software (Version 6.0, GraphPad Software Inc., San Diego, CA, USA) with the unpaired $t$-test. A $P$ value of $<0.05$ was considered statistically significant. Results are expressed as mean \pm standard deviation (SD).

\section{Results}

\subsection{FAdV-4 strain SD1511 isolated from clinical samples displaying HHS}

Virus from a suspected clinical sample exhibiting HHS was first isolated by inoculation of SPF embryonated chicken eggs with the supernatant of homogenized liver tissue samples through the chick chorioallantoic membrane. The infected embryonated eggs were small, hemorrhagic, and not fertile compared to the control eggs. Following three passages in embryonated chicken eggs, the DNA of the isolated virus was detected by PCR and confirmed by sequencing. The sequence analysis of the hexon gene demonstrated that the virus is clustered in the FAdV-C species (data not shown), with serotype 4, which was further confirmed by genome analysis (shown below). The isolate was named SD1511.

SD1511 was then inoculated into CEK cells by passage of the virus at least 10 times. The isolate was found to replicate well in CEK cells as determined by PCR and IFA. However, the isolate caused no obvious cytopathic effect in CEK cells. The TCID $_{50}$ of the isolate reached $10^{3.2} / 0.1 \mathrm{~mL}$ in CEK cells by the 10 th generation.

\subsection{High mortality in SD1511-inoculated chickens}

In all the experimental challenge groups, most of the SD1511-inoculated chickens exhibited significant 
clinical signs, including death, depression, lack of grooming, and green soft feces.

SD1511-inoculated chickens of the i.m. group exhibited the most severe clinical signs, which were observed at $2 \mathrm{dpi}$, with death starting at $3 \mathrm{dpi}$ in both the 7- and 21-d-old groups (Fig. 1a). The majority of the infected chickens died at 5-8 dpi, and the mortality rates reached $93.0 \%(13 / 14), 80.0 \%(8 / 10)$, and $100.0 \%(10 / 10)$ in the 7-, 21-, and 35-d-old groups, respectively (Fig. 1a). No birds in the control group died.

Compared with the i.m. group, chickens in the i.n. group displayed milder clinical signs after $3 \mathrm{dpi}$. The mortality of infected 7-, 21-, and 35-d-old chickens was $50.0 \%(5 / 10), 57.2 \%(8 / 14)$, and $50.0 \%$ (6/12), respectively. The chickens died at 4-10 dpi (Fig. 1b).

To compare the effects of different infectious routes on the pathogenicity of SD1511, 35-d-old chickens were infected with the same dose of SD1511 by i.m., i.n., or o.r. Similar to the results shown in Fig. 1a, chickens infected by i.m. exhibited clinical signs as early as 2 dpi and all died at 3-7 dpi (Fig. 1c). The mortality of chickens infected both by i.n. and o.r. was equivalent, $50 \%$ lower than the i.m. group, but with a longer course of disease (Fig. 1c).

\subsection{Severe gross pathology and microscopic le- sions caused by SD1511}

The animals were collected and pathological changes were observed. Various severities of liver necropsy and hydropericardium were observed in the different age and infection-route groups (Fig. 2). The longer the course of disease, the more severe the liver necrosis (Fig. 2). In the 7-d-old i.n. group, liver necrosis was observed in every infected animal, while hydropericardium was observed in only some cases (Fig. 2a). In the 21- and 35-d-old i.n. groups, mild hydropericardium was observed at 6 and $8 \mathrm{dpi}$ and severe hydropericardium at 9 dpi (Fig. 2a).

In the 35-d-old i.m. group, mild and typical hydropericardium were observed at 4 and $5 \mathrm{dpi}$, respectively (Fig. 2b). In the i.n. (Fig. 2a) and o.r. (Fig. 2b) groups, severe hydropericardium was observed later at 7 and $9 \mathrm{dpi}$, and liver necrosis appeared more severe, with the liver taking on a yellow green color, compared with the i.m. group at 7 dpi (Fig. 2).

Histopathological changes were confined to the heart and liver. Epicardial edemas were observed in
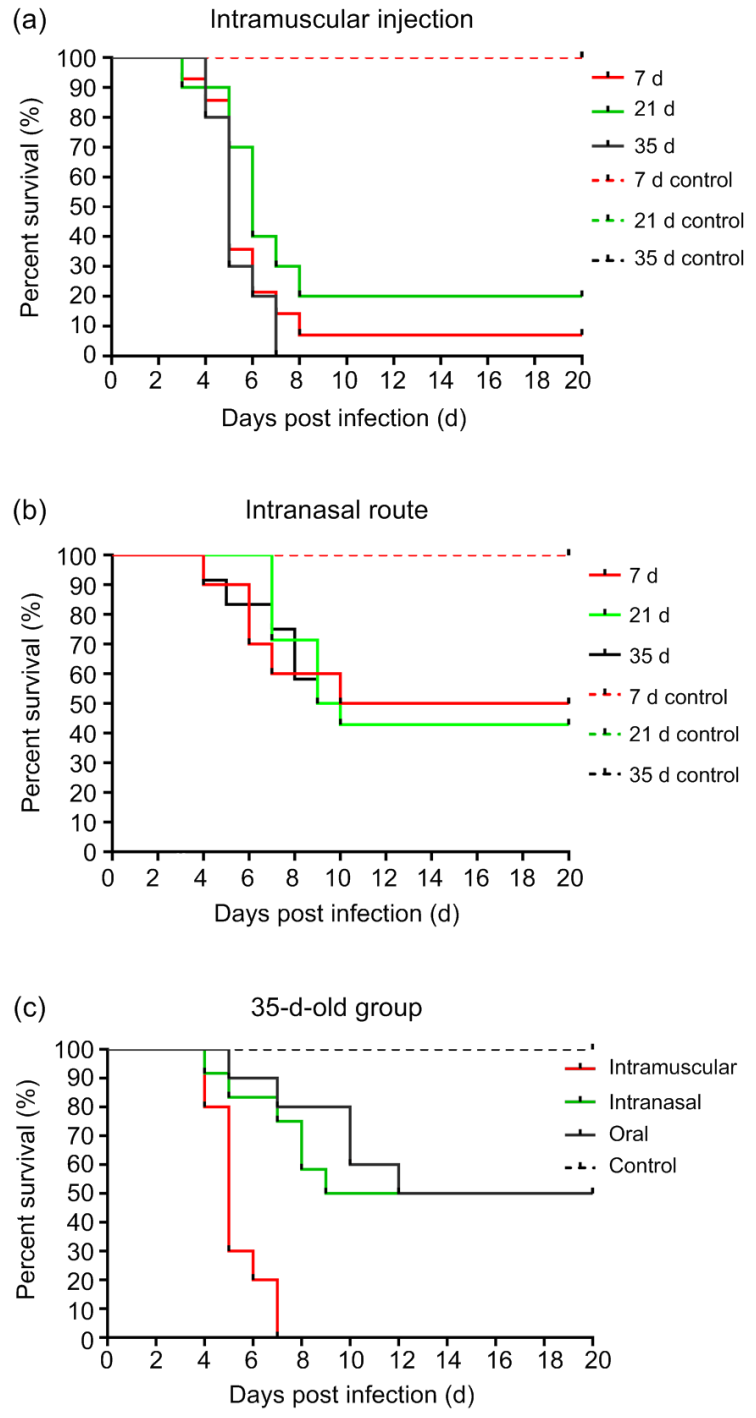

Fig. 1 Survival rates of chickens challenged with SD1511 (a) 7-, 21-, and 35-d-old SPF chickens infected by intramuscular injection. (b) 7-, 21-, and 35-d-old SPF chickens infected by intranasal route. (c) 35-d-old SPF chickens infected by all three routes, intramuscular injection, intranasal route, and oral route. No birds in control groups died

the heart tissues of most infected chickens with hydropericardium. Vacuolar degeneration with hydropic and fatty changes was observed in necrotic areas, and basophilic intranuclear inclusion bodies could be seen in hepatic cells (Fig. 3a). To confirm virus presence in the tissue of infected animals, immunohistochemistry was performed on liver sections of infected chickens in the 35-d-old i.m. group. The result indicates that a positive viral signal was widely distributed in hepatic cells (Fig. 3b). 
(a)

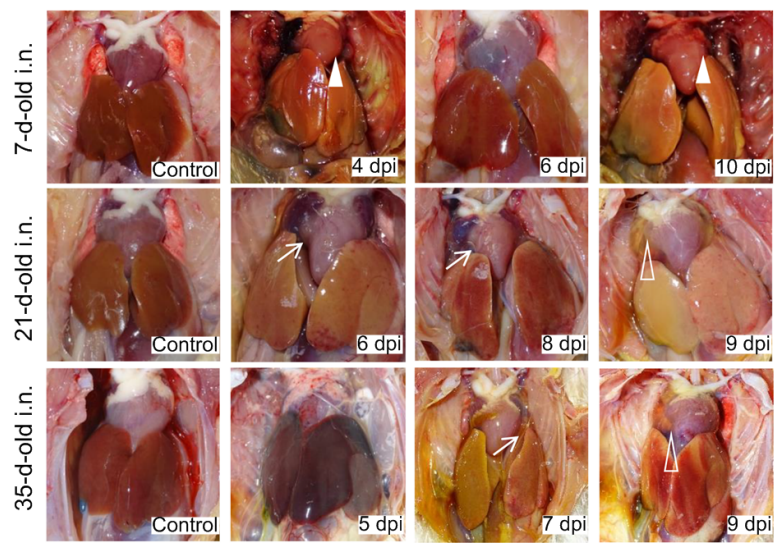

(b)

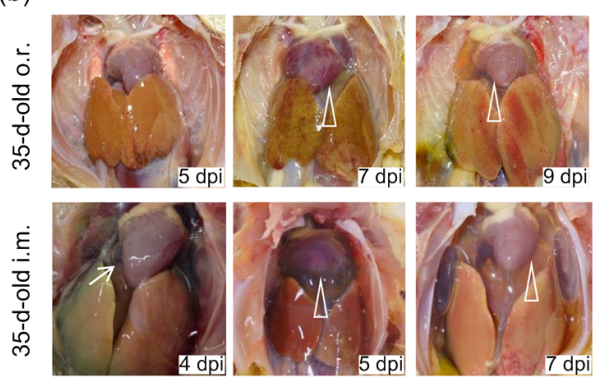

Fig. 2 Gross liver and heart lesions of SD1511-infected chickens

(a) Livers and hearts of 7-, 21-, and 35-d-old SPF chickens infected by intranasal route (i.n.); (b) Livers and hearts of 35-d-old SPF chickens infected by intramuscular injection (i.m.) and oral route (o.r.). Livers of infected chicken are swollen and faded in color. Mild hydropericardium with small liquid in the pericardial sac (solid white triangle), intermediate hydropericardium with amber colored liquid (white arrow), severe hydropericardium with amber colored liquid (white hollow triangle) were observed

\subsection{The highest viral load in the livers of infected chickens}

The levels of viral DNA expression in different tissues of the 35-d-old group are summarized in Fig. 4. For all three infection routes, viral DNA expression levels in livers were significantly higher than those in other tissues, including the hearts, lungs, and kidneys, with the exception of the viral load in the kidneys of birds in the i.m. group (Fig. 4). The difference in the level of viral DNA in the liver compared with other tissues in the o.r. and i.n. groups $(P<0.01)$ was more significant than that in the i.m. group $(P<0.05$; Fig. 4). The level of viral DNA in the hearts, (a) Heart
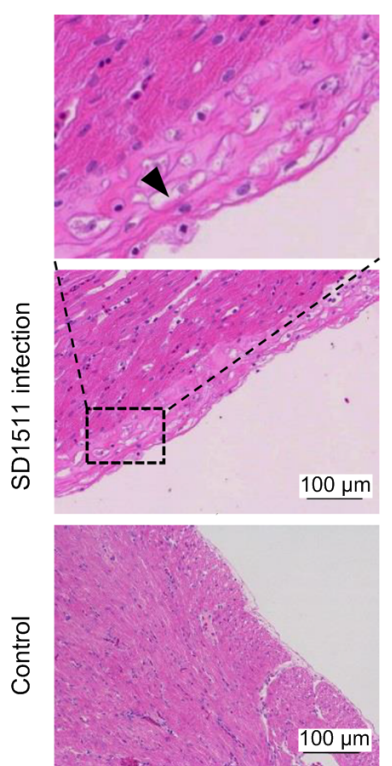

(b)

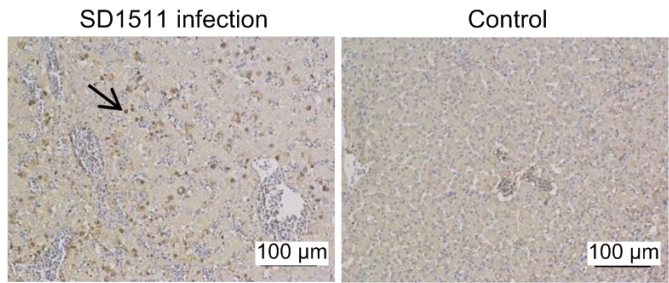

Fig. 3 Histopathological changes of hearts and livers of SD1511-infected chickens

(a) The heart showed obvious epicardial edema, epicardial cell enlargement, and vacuolization (black triangle). Hepatic cells presented with intranuclear inclusion bodies (black arrows) and vacuolar degeneration including hydropic and fatty change (hollow triangle); (b) Immunohistochemistry examinations of liver. Black arrow indicates positive signals for viral particles in the liver cells. Scale bar $=100 \mu \mathrm{m}$

lungs, and kidneys of infected animals was not significantly different, except between the hearts and lungs of the o.r. group (Fig. 4).

\subsection{Long shedding period following virus infection}

As shown in Table 1, infected chickens of all three age groups in the i.n. group started shedding virus at 2 dpi, and kept a high shedding rate (100.0\%) up to $17 \mathrm{dpi}$. The shedding rate then decreased gradually, reaching zero at $40 \mathrm{dpi}$ in the 7-d-old group, with a few birds still positive at 40 dpi in the 21 - and 35-d-old groups (Table 1). Viral DNA was detected in both oropharyngeal swabs and cloacal swabs by PCR. 
Table 1 Virus shedding of 7-, 21-, and 35-d-old chickens-infected by intranasal route

\begin{tabular}{clcccccccc}
\hline \multirow{2}{*}{ Group } & \multirow{2}{*}{ Sample } & \multicolumn{7}{c}{ Shedding rate (positive number/total number) } \\
\cline { 3 - 9 } & & $2 \mathrm{dpi}$ & $7 \mathrm{dpi}$ & $12 \mathrm{dpi}$ & $17 \mathrm{dpi}$ & $25 \mathrm{dpi}$ & $30 \mathrm{dpi}$ & $35 \mathrm{dpi}$ & $40 \mathrm{dpi}$ \\
\hline \multirow{2}{*}{$\mathrm{d} \mathrm{d}$} & Oropharyngeal & $10 / 10$ & $7 / 7$ & $5 / 5$ & $5 / 5$ & $3 / 5$ & $1 / 5$ & $1 / 5$ & $0 / 5$ \\
& Cloacal & $10 / 10$ & $7 / 7$ & $5 / 5$ & $5 / 5$ & $4 / 5$ & $1 / 5$ & $2 / 5$ & $0 / 5$ \\
$\mathrm{*} \mathrm{d}$ & Oropharyngeal & $14 / 14$ & $14 / 14$ & $6 / 6$ & $6 / 6$ & $5 / 6$ & $4 / 6$ & $2 / 6$ & $1 / 6$ \\
& Cloacal & $14 / 14$ & $14 / 14$ & $6 / 6$ & $6 / 6$ & $5 / 6$ & $5 / 6$ & $1 / 6$ & $1 / 6$ \\
& Oropharyngeal & $12 / 12$ & $10 / 10$ & $6 / 6$ & $6 / 6$ & $5 / 6$ & $3 / 6$ & $2 / 6$ & $0 / 6$ \\
& Cloacal & $12 / 12$ & $10 / 10$ & $6 / 6$ & $6 / 6$ & $5 / 6$ & $4 / 6$ & $2 / 6$ & $1 / 6$ \\
\hline
\end{tabular}

dpi: day post-inoculation

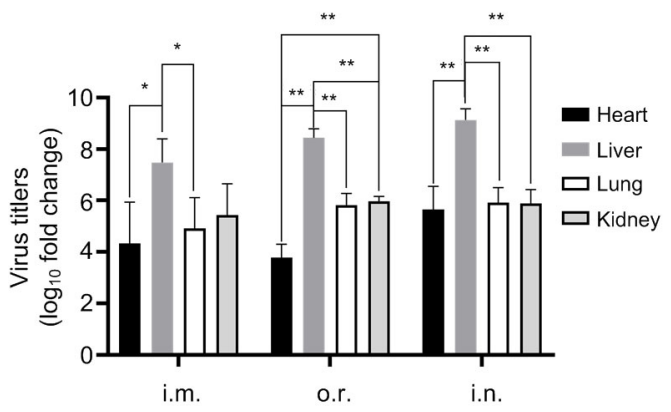

Fig. 4 Viral loads in tissues of 35-d-old group treated by different routes

Values are expressed as mean $\pm \mathrm{SD}$ and significance was evaluated by unpaired $t$-tests using the Holm-Sidak method. ${ }^{*} P<0.05,{ }^{* *} P<0.01$. i.m.: intramuscular injection; i.n.: intranasal route; o.r.: oral route

\subsection{Neutralization activity of infected chicken's serum to SD1511}

Following incubation with serial dilutions $\left(10^{-1}\right.$, $10^{-2}$, and $10^{-3}$ ) of sera from surviving SD1511infected chickens, virus was inoculated into CEK cells and subsequently passed to the second generation. No viral DNA was detected from the second generation by both real-time PCR and common PCR, although the specific viral DNA was detected in the negative serum control group and PBS control group (Figs. 5a and 5b). To determine the neutralization titer of the sera, CEK cells infected with pre-incubated virus were detected by IFA with antiserum to the hexon protein of FAdV-4. The specific fluorescence was only observed in the $10^{-4}, 10^{-5}, 10^{-6}, 10^{-7}$ dilution groups and in the PBS control group, but not in the $10^{-2}$ or $10^{-3}$ dilution group. Figs. $5 \mathrm{c}$ and $5 \mathrm{~d}$ demonstrated the IFA detection of CEK cells in $10^{-3}$ and $10^{-4}$ dilution groups. The results indicate that the sera from SD1511-infected chicken have neutralization activity, the neutralization titer of the sera being around $10^{3.33}$.

\subsection{Distinct differences of the genome of SD1511 from overseas strains}

The full length of the SD1511 genome, $43724 \mathrm{bp}$, was completely sequenced (GenBank Accession No. MF496037). Phylogenetic analysis revealed that strain SD1511 is classified as serotype 4 of the FAdV-C group, together with reference strains KR5 (HE608152.1) and ON1 (GU188428), as well as the following selected field strains recently isolated in China: HLJFAd15 (KU991797.1), CH/SXCZ/2015 (KU558762.1), HB1510 (KU587519.1), and JSJ13 (KM096544.1) (Fig. 6). In the phylogenetic tree, SD1511 was of some distance from different FAdV strains in other FAdV groups (Fig. 6). The genomic identities between SD1511 and the strains of FAdV-A, FAdV-B, FAdV-D, and FAdV-E were around 38.6\%, $39.9 \%, 39.1 \%$, and $49.7 \%$, respectively (data not shown).

The strains isolated in China (SD1511, HLJFAd15, HB1510, JSJ13, and CH-SXCZ-2015) share high identities, ranging from $99.9 \%$ to $100 \%$; and the strains isolated outside of China (ON1, KR5, MXSHP95, and B1-7) share high gene nucleotide identities ranging from $97.7 \%$ to $98.9 \%$. However, lower identities were observed ranging from $93.6 \%-94.6 \%$ between the overseas strains (ON1, KR5, MX-SHP95, and B1-7) and the domestic strains (SD1511, HLJFAd15, HB1510, JSJ13, and CH-SXCZ-2015) (Table S2).

Compared with the overseas strains KR5, ON1, B1-7, and MX-SHP95, all strains reported in China, including SD1511, have a 10-bp insertion in tandem repeat region $\mathrm{B}$ (TR-B), a 3-bp insertion in the $55 \mathrm{kDa}$ protein-coding sequence, and a 6-bp deletion in the $33 \mathrm{kDa}$ protein-coding fragment. Furthermore, there is a 1966-bp deletion in the genome of Chinese isolates, which results in the absence of almost the entire open reading frame (ORF) 19, the entire ORF27, and 
(a)

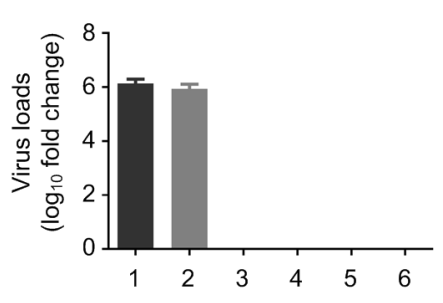

(c)

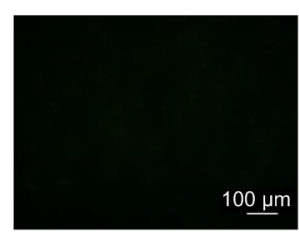

(b) M 123456

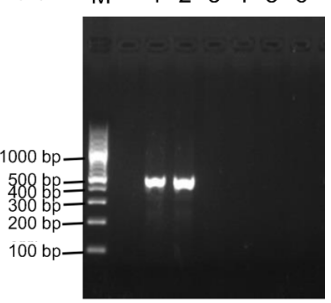

(d)

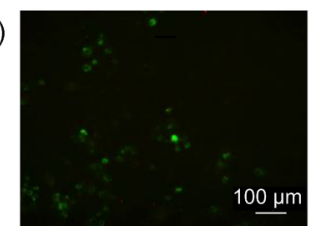

Fig. 5 Neutralization activity of sera from SD1511infected chickens

$(a, b)$ Detection of viral DNA by real-time PCR (a) and common PCR (b). SD1511 was pre-incubated with serial dilutions of sera of SD1511-infected chickens at $45 \mathrm{dpi}$, then inoculated into CEK cells and passed to CEK cells for a second time. The DNA of the culture of the second generation was detected. M, marker; 1, PBS control; 2, uninfected serum in $10^{-1}$ dilution; 3-5, SD1511-infected chicken sera in $10^{-1}, 10^{-2}$, and $10^{-3}$ dilutions, respectively; 6 , blank control (without sera or virus). The values of realtime PCR detection are expressed as mean \pm SD. (c, d) Detection of infected CEK cells by IFA. The CEK cells were infected with SD1511 pre-incubated with sera of infected chickens in $10^{-3}$ (c) and $10^{-4}$ (d) dilutions. The signals were detected with anti-serum to hexon of FAdV-4

the entire ORF48 (Fig. 7). In the GA repeat region between genes $p X$ and $p V I$, all the Chinese isolates, including SD1511, have longer GA repeats than those of the overseas strains MX-SHP95, KR5, B1-7, and ON1.

Many differences between the Chinese and oversea strains were also observed in the TC repeat region between the protease and the DNA-binding protein (DBP) coding sequence, TR-E, the region between TR-E and ORF19A, and the 6.2-kDa coding region as shown in Fig. 7.

Specifically, a 33-bp insertion was observed in the ORF29 of the first isolated FAdV-4 strain JSJ13 compared with other isolates, while a 33-bp deletion present in the ON1 strain was observed in the related region (Fig. 7 and Table 2).

Variations were also observed in the amino acid sequences of the structural proteins of FAdV-4 (Table 2). Similar to many highly virulent FAdV-4, SD1511 has the same amino-acid substitutions at position 42 ( $\mathrm{S}$ to $P$ ) in the penton base protein, position 432 ( $S$ to $G$ ) in the fiber 1 protein, position 219 (G to D), 300 (I to T), 305 (S to A), 307 (P to A), 378 (I to T), 380 (A to T), 435 ( $\mathrm{T}$ to $\mathrm{S}$ ), and 453 ( $\mathrm{S}$ to $\mathrm{A}$ ) in the fiber 2 protein compared with the reference strains KR5 and ON1 (Table 2) (Liu et al., 2016). However, position 42 in the penton base protein of the mildly pathogenic

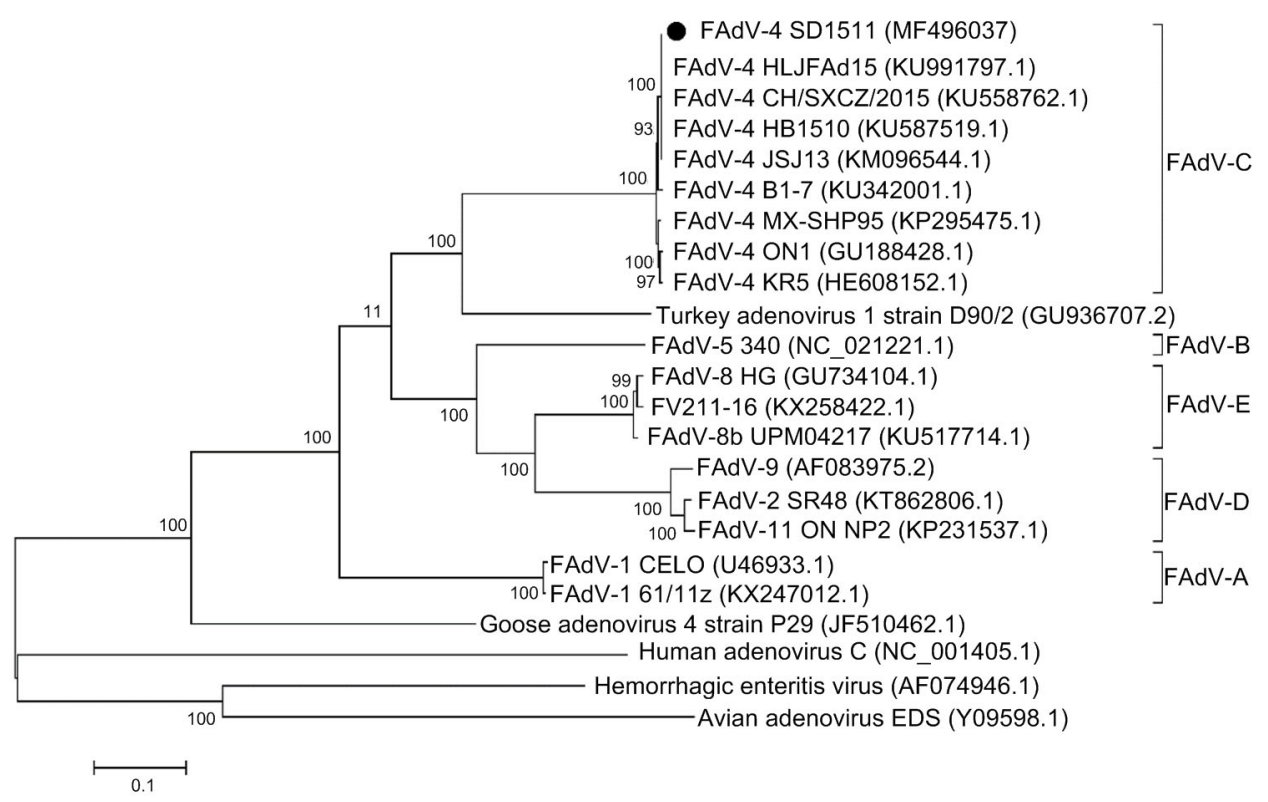

Fig. 6 Phylogenetic tree based on the complete genome of avian adenoviruses belonging to genera Aviadenovirus Phylogenetic tree was constructed using the neighbor-joining analysis with 500 bootstrap replicates, maximum composite likelihood method in MEGA5.05 software. SD1511 isolated in this study is indicated $(\bullet)$ 


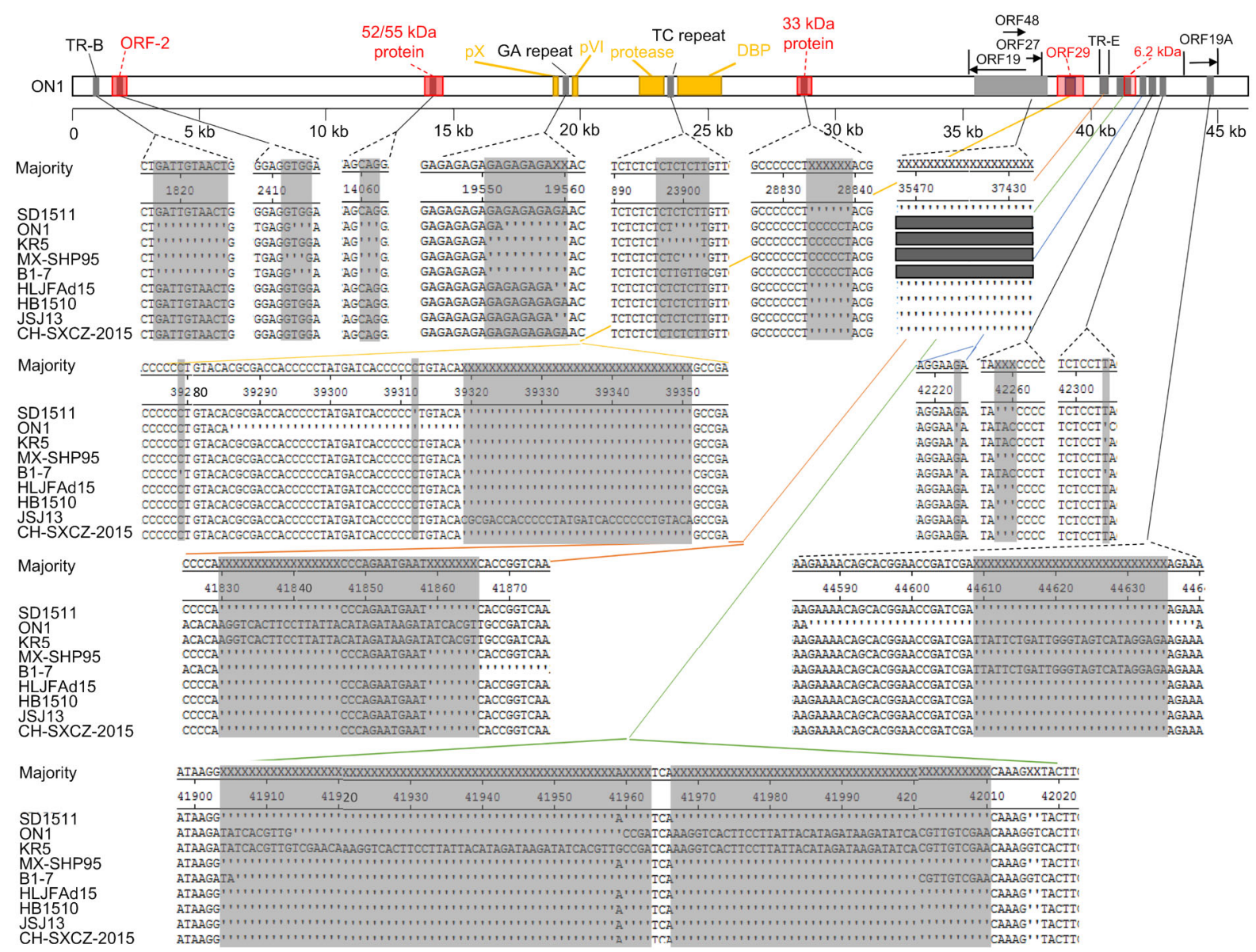

Fig. 7 Comparison of the complete genomes of FAdV-4 strain

Significant mutations are displayed. The red regions indicate the coding sequence (CDS) regions where changes in the amino sequence are observed. The yellow regions indicate the CDS region alongside the deletions/insertions. The gray regions indicate the changes observed (Note: for interpretation of the references to color in this figure legend, the reader is referred to the web version of this article)

strain B1-7 also exhibits a change from S to P. P42 may not affect virulence or is less significant than other defined amino acids. According to a previous report (Park et al., 2017), A380 in fiber 2 may also have less effect on virulence.

\section{Discussion}

Fowl adenovirus is an important genus of Adenoviridae, and includes five species (A-E) with 12 serotypes. Infection of chickens with FAdV-4 of species $\mathrm{C}$ causes severe inclusion body hepatitis (IBH) and hydropericardium syndrome (HPS), leading to large economic losses in China since 2013.

One FAdV-4 strain, SD1511, was isolated from clinical samples showing IBH and HPS from
Shandong Province, China, in 2015. The virus was first thought to propagate in SPF embryonated chicken eggs via the chorioallantoic membrane and cause pathogenicity as described in a previous report (Schat and Purchase, 1989). However, following three passages in embryonated eggs, the isolates could not be detected in culture by PCR. According to an earlier report, CEK cells are suitable for propagating FAdVs (Chomiak et al., 1961), and we successfully infected CEK cells with SD1511 achieving titers of $10^{3.2} \mathrm{TCID}_{50} / 0.1 \mathrm{~mL}$ on the 10th passage. However, unlike CELO virus, SD1511 did not induce characteristic cytopathic effect (CPE) in CEK cells (Chomiak et al., 1961). Most FAdVs are reported to grow well in chicken embryo liver (CEL) cells or chicken hepatoma LMH cells (Alexander et al., 1998; Grgić et al., 2011; Li et al., 2016) with FAdV-4 
Table 2 Variation of the SD1511 genome as compared to FAdV-4 reference strains

\begin{tabular}{|c|c|c|c|c|c|c|}
\hline \multirow{2}{*}{ Variation } & \multirow{2}{*}{ Location $^{*}$} & \multicolumn{5}{|c|}{ Selected reference strains } \\
\hline & & SD1511 & MX-SHP9 & ON1 & KR5 & $\mathrm{B} 1-7$ \\
\hline 10-bp insertion & TR-B & + & - & - & - & - \\
\hline 3-bp insertion & ORF-2 & + & - & - & + & - \\
\hline 3-bp insertion & $52 / 55 \mathrm{kDa}$ & + & - & - & - & - \\
\hline 6-bp deletion & $33 \mathrm{kDa}$ & + & - & - & - & - \\
\hline 1966-bp deletion & $35389-37354 \mathrm{bp}$ & + & - & - & - & - \\
\hline 33-nt deletion & C-terminal of ORF29 & + & + & + & + & + \\
\hline Longer GA repeat & Between $p X$ and $p V I$ genes & + & - & - & - & - \\
\hline Longer TC repeat & $23855-23864$ bp & + & + & - & - & + \\
\hline TR-E deletion & $41749-41762$ bp; 41766-41809 bp & + & + & + & - & + \\
\hline 1-bp insertion & After $42021 \mathrm{bp}$ & + & + & - & - & - \\
\hline 3-bp deletion & $2056-42058 \mathrm{bp}$ & + & + & - & - & - \\
\hline 1-bp insertion & After $42100 \mathrm{bp}$ & + & + & - & - & - \\
\hline $\mathrm{S} 432 \mathrm{G}$ & Fiber 1 protein & + & + & - & - & - \\
\hline G219D & Fiber 2 protein & + & + & - & - & - \\
\hline $\mathrm{I} 300 \mathrm{~T}$ & Fiber 2 protein & + & + & - & - & - \\
\hline $\mathrm{S} 305 \mathrm{~A}$ & Fiber 2 protein & + & + & - & - & - \\
\hline P307A & Fiber 2 protein & + & + & - & - & - \\
\hline $\mathrm{I} 378 \mathrm{~T}$ & Fiber 2 protein & + & + & - & - & - \\
\hline A380T & Fiber 2 protein & + & + & - & - & - \\
\hline $\mathrm{T} 435 \mathrm{~S}$ & Fiber 2 protein & + & + & - & - & - \\
\hline $\mathrm{S} 453 \mathrm{~A}$ & Fiber 2 protein & + & + & - & - & - \\
\hline $\mathrm{S} 42 \mathrm{P}$ & Penton base protein & + & + & - & - & + \\
\hline
\end{tabular}

"The locations refer to the genome sequence of ON1 (GenBank accession No. GU188428). "+" means that variation was present, while "_" means that the variation did not exist

propagating better in LMH than in CEL cells and yielding 100-fold higher virions (Alexander et al., 1998). Our data also showed that the liver carried a higher viral load than the heart, lung, or kidney of infected chickens (Fig. 4). Therefore, it is understandable that SD1511 only reached $10^{3.2} \mathrm{TCID}_{50} /$ $0.1 \mathrm{~mL}$ in CEK cells. Despite this finding, CEK cells provide a candidate cell line for $\mathrm{FAdV}-4$ propagation and vaccine development since SD1511 cultured in CEK causes typical HHS and high mortality to infected chickens (Figs. 1 and 2).

High virulence of FAdV-4 was commonly found in Chinese isolates. Infection with JSCZ15, SD15, and SDWF15 isolates results in $80 \%-100 \%$ mortality (Ye et al., 2016). Consistent with a previous report (Mazaheri et al., 1998), the pathogenicity of FAdV-4 isolate was related to the infection route. HN/151025 caused $90 \%$ mortality to chickens by i.m. infection and $30 \%$ by o.r. infection (Li et al., 2016), while o.r. infection with JSJ13 led to a $28.6 \%$ death rate (Zhao et al., 2015). Most previous studies only considered two common infection routes, i.m. and o.r. By contrast, we compared pathogenicity in 7-, 21-, and 35-d-old chickens using three different challenge routes, i.m., i.n., and o.r. The results indicated that i.m. was the most sensitive route for FAdV-4 infection, which is consistent with previous studies (Mazaheri et al., 1998; Li et al., 2016). We also found that infection of FAdV-4 by i.n. caused high mortality (up to $50 \%$ ), higher even than infection by o.r. Taking into account the data from our study with evidence that FAdV can cause quail bronchitis (Bose and Grumbles, 1959), we suspect that the upper respiratory tract as well as the digestive tract is a sensitive organ for FAdV infection. Another piece of evidence to support this speculation is the detection of FAdV-4 in the oropharyngeal swabs of infected chicken, suggesting that air-borne transmission may be a major transmission route for FAdV-4 infection.

The results of animal experiments demonstrate that birds of all ages were infected by SD1511, resulting in high mortality. This is consistent with a 
previous report that 1-5-week-old chickens are sensitive to FAdV-4, with the severity of infection and mortality increasing with chicken age (Mazaheri et al., 1998; Li et al., 2017). We also report that viral load was highest in the liver in all infected groups, but with the difference in viral load between the liver and other tissues being less significant in the i.m. group (Fig. 4). Whether this suggests that infection by i.m. is more evenly distributed and directed to different organs, thus leading to higher mortality, needs to be further investigated.

In the pathogenicity experiments, HHS was observed in all the infected chickens, with 35-d-old chickens showing more typical pathological changes than 7- and 21-d-old chickens (Figs. 2a and 2b). This might be related to longer disease course in the older chickens. It was reported that most of the clinical FAdV-4 infection cases have been observed in 3-5-week-old broilers (Jaffery, 1988; Mase et al., 2012; Zhao et al., 2015). However, it is unclear why the 35-d-old chickens seemed more sensitive to FAdV-4 than the younger chickens.

Viral DNA from oropharyngeal and cloacal swabs of infected chickens was detected from 2 to $40 \mathrm{dpi}$, indicating the long period of virus shedding. To our surprise, we found that the serum from infected chickens completely neutralized SD1511 virus in fresh cell cultures. According to the report by $\mathrm{Li}$ et al. (2016), seroconversion was detected in infected chickens as early as $7 \mathrm{dpi}$ and reached its peak from 14 to $28 \mathrm{dpi}$, implying that the virus can survive for a long time in the presence of high antibody and keep shedding for up to $40 \mathrm{~d}$ (in our study). The safety and efficacy of vaccine strategies against FAdV-4 is a challenge.

Since 2013, FAdV-4 has become widely distributed in China, and numerous deletions and mutations have occurred in the viral genome (Zhao et al., 2015; Liu et al., 2016). SD1511 displays more than 99.9\% identity with FAdV-4 strains isolated recently in China, and contains many similar deletions/ insertions to the Chinese isolates, including a 6-bp deletion in TR-B and 33-kDa coding sequence, a 3-bp insertion in 52/55-kDa coding sequence, a 1966-bp deletion between positions 35389 and 37354 referred to as the sequence of $\mathrm{ON} 1$, and also the longer GA repeat. These were not found in the corresponding regions of overseas strains ON1, KR5, B1-7, and
MX-SHP95. This suggests that the genome of FAdV-4 circulating in China is different from overseas strains. However, it is still unclear that these deletions/ insertions are related to the recent HHS outbreaks in China. The long 1966-bp deletion observed in Chinese FAdV-4 isolates leads to the deletion of ORF19, ORF27, and ORF48, indicating that these ORFs are not necessary for FAdV-4 replication.

When comparing the sequences between pathogenic and non-pathogenic strains, we found that the deletion/insertion patterns in the TR-E, ORF19A, and the regions between TR-E and ORF19A (Fig. 6 and Table 2), as well as some mutations listed in Table 2, were different. Further experiments are necessary to find out the key sequences responsible for FAdV-4 pathogenicity.

In the genome analysis of FAdV-4, many differences were also found, even between the two nonpathogenic strains ON1 and KR5. For example, a 3-bp insertion is present in the ORF2 of KR5, but not in ON1; a 33-bp deletion is present in the ORF29 of $\mathrm{KR} 5$, but not in ON1; a longer deletion in the ORF19A of ON1 is not present in the ORF19A of KR5 (Fig. 6 and Table 2). Whether these deletions are related to pathogenicity or virus replication of FAdV-4 needs to be further investigated.

\section{Conclusions}

We isolated one FAdV-4 strain, SD1511, and adapted it to CEK cells. Mortality amongst 7-35-dold chickens infected with SD1511 by i.m., i.n., and o.r. was $50 \%-100 \%$. Infection was more severe in the 35-d-old than in the 7- and 21-d-old chickens, and the mortality was highest in the intramuscular injection group. SD1511 induced high neutralization antibody in infected chickens. The genome of SD1511 contained many deletions and mutations consistent with other FAdV-4 strains isolated in China, but with distinct variations compared with overseas reference strains. The findings in this study suggest that SD1511 might be used as a prototype for the study of pathogenesis and vaccine development.

\section{Contributors}

Kai-kun MO performed the experimental research and data analysis, wrote and edited the manuscript. Chen-fei LYU, 
Shang-shang CAO, and Yan YAN performed the pathogenicity experiments and data analysis. Xia LI performed the sequence analysis and antiserum preparation. Gang XING collected the clinical samples. Min LIAO, Ji-yong ZHOU, and Xiao-juan ZHENG designed the study and edited the manuscript.

\section{Acknowledgments}

We thank Professor Guo-zhong ZHANG (Key Laboratory of Animal Epidemiology and Zoonosis, Ministry of Agriculture, College of Veterinary Medicine, China Agricultural University, Beijing, China) for providing chicken anti-FAdV hyperimmune serum.

\section{Compliance with ethics guidelines}

Kai-kun MO, Chen-fei LYU, Shang-shang CAO, Xia LI, Gang XING, Yan YAN, Xiao-juan ZHENG, Min LIAO, and Ji-yong ZHOU declare that they have no conflict of interest.

All institutional and national guidelines for the care and use of laboratory animals were followed.

\section{References}

Alexander HS, Huber P, Cao J, et al., 1998. Growth characteristics of fowl adenovirus type 8 in a chicken hepatoma cell line. J Virol Methods, 74(1):9-14. https://doi.org/10.1016/s0166-0934(98)00062-7

Bose RTD, Grumbles LC, 1959. The relationship between quail bronchitis virus and chicken embryo lethal orphan virus. Avian Dis, 3(3):321-344. https://doi.org/10.2307/1587679

Choi KS, Kye SJ, Kim JY, et al., 2012. Epidemiological investigation of outbreaks of fowl adenovirus infection in commercial chickens in Korea. Poult Sci, 91(10):25022506. https://doi.org/10.3382/ps.2012-02296

Chomiak TW, Luginbuhl RE, Helmboldt CF, 1961. Tissue culture propagation and pathology of celo virus. Avian Dis, 5(3):313-320. https://doi.org/10.2307/1587640

Ganesh K, Raghavan R, 2000. Hydropericardium hepatitis syndrome of broiler poultry: current status of research. Res Vet Sci, 68(3):201-206. https://doi.org/10.1053/rvsc.1999.0365

Grgić H, Yang DH, Nagy É, 2011. Pathogenicity and complete genome sequence of a fowl adenovirus serotype 8 isolate. Virus Res, 156(1-2):91-97. https://doi.org/10.1016/j.virusres.2011.01.002

Hess M, 2000. Detection and differentiation of avian adenoviruses: a review. Avian Pathol, 29(3):195-206. https://doi.org/10.1080/03079450050045440

Jaffery MS, 1988. A treatise on angara disease (hydropericardiumpulmonary oedema-hepatonephritis syndrome). J Pak Vet Med Assoc, 34:1-33.

Kaján GL, Kecskeméti S, Harrach B, et al., 2013. Molecular typing of fowl adenoviruses, isolated in hungary recently, reveals high diversity. Vet Microbiol, 167(3-4):357-363. https://doi.org/10.1016/j.vetmic.2013.09.025
Li HX, Wang J, Qiu LY, et al., 2016. Fowl adenovirus species C serotype 4 is attributed to the emergence of hepatitishydropericardium syndrome in chickens in China. Infect Genet Evol, 45:230-241. https://doi.org/10.1016/j.meegid.2016.09.006

Li HY, Yin YB, Guo YY, et al., 2010. Isolation and PCR identification of 12 strains of inclusion body hepatitis virus from clinical broilers. Vet Sci China, 40(7):722-727 (in Chinese). https://doi.org/10.16656/j.issn.1673-4696.2010.07.013

Li PH, Zheng PP, Zhang TF, et al., 2017. Fowl adenovirus serotype 4: epidemiology, pathogenesis, diagnostic detection, and vaccine strategies. Poult Sci, 96(8):2630-2640. https://doi.org/10.3382/ps/pex087

Liu YK, Wan WY, Gao DS, et al., 2016. Genetic characterization of novel fowl aviadenovirus 4 isolates from outbreaks of hepatitis-hydropericardium syndrome in broiler chickens in China. Emerg Microbes Infect, 5(1):1-8. https://doi.org/10.1038/emi.2016.115

Lund S, Holman GD, Schmitz O, et al., 1995. Contraction stimulates translocation of glucose transporter GLUT4 in skeletal muscle through a mechanism distinct from that of insulin. Proc Natl Acad Sci USA, 92(13):5817-5821. https://doi.org/10.1073/pnas.92.13.5817

Mase M, Nakamura K, Minami F, 2012. Fowl adenoviruses isolated from chickens with inclusion body hepatitis in Japan, 2009-2010. J Vet Med Sci, 74(8):1087-1089. https://doi.org/10.1292/jvms.11-0443

Mazaheri A, Prusas C, Voß M, et al., 1998. Some strains of serotype 4 fowl adenoviruses cause inclusion body hepatitis and hydropericardium syndrome in chickens. Avian Pathol, 27(3):269-276. https://doi.org/10.1080/03079459808419335

Mittal D, Jindal N, Tiwari AK, et al., 2014. Characterization of fowl adenoviruses associated with hydropericardium syndrome and inclusion body hepatitis in broiler chickens. VirusDisease, 25(1):114-119. https://doi.org/10.1007/s13337-013-0183-7

Ojkic D, Martin E, Swinton J, et al., 2008. Genotyping of Canadian isolates of fowl adenoviruses. Avian Pathol, 37(1):95-100. https://doi.org/10.1080/03079450701805324

Park HS, Lim IS, Kim SK, et al., 2017. Molecular analysis of the hexon, penton base, and fiber-2 genes of Korean fowl adenovirus serotype 4 isolates from hydropericardium syndrome-affected chickens. Virus Genes, 53(1):111-116. https://doi.org/10.1007/s11262-016-1393-z

Schachner A, Matos M, Grafl B, et al., 2018. Fowl adenovirusinduced diseases and strategies for their control - a review on the current global situation. Avian Pathol, 47(2):111126. https://doi.org/10.1080/03079457.2017.1385724

Schat K, Purchase HG, 1989. A Laboratory Manual for the Isolation and Identification of Avian Pathogens, 3rd Ed. American Association of Avian Pathologists, Kennett Square, PA, American, p.176-181.

Ye JQ, Liang GC, Zhang JJ, et al., 2016. Outbreaks of serotype 
4 fowl adenovirus with novel genotype, China. Emerg Microbes Infect, 5(1):1-12.

https://doi.org/10.1038/emi.2016.50

Zhao J, Zhong Q, Zhao Y, et al., 2015. Pathogenicity and complete genome characterization of fowl adenoviruses isolated from chickens associated with inclusion body hepatitis and hydropericardium syndrome in China. PLoS ONE, 10(7):e0133073.

https://doi.org/10.1371/journal.pone.0133073

\section{List of electronic supplementary materials}

Table S1 Primers used to amplify the complete genomic sequence of FAdV-4 strain SD1511

Table S2 Genome sequence identities (\%) among SD1511 and FAdV-4 reference strains

\section{中文概要}

\section{题 目: 禽腺病毒 4 型 (FAdV-4) 分离株对鸡的致病力 及其基因组分析}

目 的: 了解禽腺病毒 4 型 (FAdV-4) 分离株在口服、滴 鼻点眼和肌注三种感染途径下对 7 35 日龄鸡的 致病性, 并分析该分离株与其他参考毒株在基因 组水平上的差异。
创新点: 用原代鸡胚肾细胞 (CEK) 成功培养了 FAdV-4 分离株。发现除了常用的口服和肌注途径, FAdV-4 也可通过滴鼻点眼接种途径感染 7、21 和 35 日龄鸡。肌注方法最为敏感, 导致感染鸡 $100 \%$ 死亡; 35 日龄鸡较 7 和 21 日龄鸡表现出更 明显的临床症状和剖检病变。

方 法: 通过口服、滴鼻点眼和肌注三种途径将 FAdV-4 分离株的 CEK 细胞培养物接种 7 35 日龄的无特 定病原体 (SPF) 鸡。通过临床症状观察、排毒 检测、病理解剖、免疫组化和组织中毒载量测定 来判断分离株的致病性及病毒在组织脏器中的分 布情况。通过病毒中和试验检测感染存活鸡的血 清中和抗体。通过全基因组测序分析, 比较 FAdV-4 分离株与国内外参考毒株的基因组序列差异。

结 论: 本研究分离鉴定的 FAdV-4 分离株 SD1511 可以 在 CEK 细胞上适应, 并可以通过口服、滴鼻点 眼和肌注三种感染途径引起 7 35 日龄的 SPF 鸡 $50 \% \sim 100 \%$ 的死亡, 其中肌注最为敏感, 死亡率 达 $100 \%$ 。感染鸡持续排毒至感染后的 40 天, 产 生具有明显中和作用的中和抗体。全基因组测序 分析结果显示, SD1511 基因组与最近国内分离 的 FAdV-4 具有很高的同源性, 但与国外的分离 株有明显的差异。

关键词：禽腺病毒 4 型（FAdV-4）分离株; 致病性; 感染 途径; 中和活性; 基因组测序分析 